\title{
Supporting Collaborative Operations within a Coalition Personnel Recovery Center
}

\author{
Gerhard Wickler, Austin Tate, and Jeffrey Hansberger
}

\begin{abstract}
I-X is a framework that can be used to create an application in which multiple agents adopt a task-centric view of a situation, and which supports the necessary coordination of their activities to respond to that situation. The I-X Process Panel provides the functionality of a to-do list and instant messaging and thus, it is a useful tool when it comes to organizing the response to an emergency. However, I-X goes well beyond this metaphor and provides a number of useful extensions that facilitate the finding and adaptation of plans for teams to respond in dynamic situations.

In the Co-OPR (Collaborative Operations for Personnel Recovery) project, the I-X framework has been used to support training exercises for personnel recovery. This paper will describe some of the initial findings that are the result of experiments conducted to evaluate the suitability and extent to which personnel recovery trainees and trainers can be supported by I-X in so-called "Command Post Exercises". The result shows that an I-X application can be useful in such a scenario by eliminating some of the basic problems that often occur.
\end{abstract}

Index Terms-Decision-making, Emergency Response, Search and Rescue, Personnel Recovery, Planning, Collaboration

\section{INTRODUCTION}

Personnel Recovery (PR) is the sum of military, diplomatic and civil efforts to affect the recovery and reintegration of isolated personnel. During any military operation Coalition or Joint Force Commanders and Staff are responsible for being prepared to accomplish the PR execution tasks throughout a specified operational area or determine and accept the risk of not doing so [4]. In order to be prepared, the USJFCOM/JPRA Personnel Recovery Education and Training Center (PRETC) trains military personnel in the execution of PR tasks. This training consists of classroom sessions in which the necessary knowledge is taught, and a series of Command Post Exercises (CPX) in which the students have to perform PR tasks in a simulated fictitious military operation called "Operation Able Sword".

One of the aims of the Co-OPR project is to evaluate the possibility of using the I-X framework to create an application that can be used to support the PR task. In this paper we shall briefly describe the I-X framework including its principal user

G. Wickler and A. Tate are with the Artificial Intelligence Applications Institute, University of Edinburgh, Edinburgh, EH8 9LE, Scotland (+44 131 6502732; fax: +44 131 6506513; e-mail: g.wickler/a.tate@ed.ac.uk).

J. Hansberger is with USJFCOM/J9, Norfolk, VA, USA (e-mail: Jeff.Hansberger@je.jfcom.mil). interface, the I-X Process Panel and underlying ontology, $<\mathrm{I}-\mathrm{N}-\mathrm{C}-\mathrm{A}>$. The Co-OPR application that was developed using the I-X framework is based on requirements that were captured during the observation of a CPX, also described in this paper. How these requirements translated into features of the application will be described next. Finally, we shall describe the results of several experiments that have taken place in AIAI's experimental Emergency Response Coordination Center (e-RCC) and at USJFCOM/J9 to evaluate the Co-OPR application for PR.

\section{THE I-X FRAMEWORK}

I-X is a framework that can be used to create an application in which multiple agents, be they human or software, adopt a task-centric view of a situation, and which supports the necessary coordination of their activities to respond to that situation. The I-X Process Panel provides the functionality of a to-do list and thus, it is a useful tool when it comes to organizing the response to an emergency. The idea of using a to-do list as a basis for a distributed task manager is not new [5]. However, I-X goes well beyond this metaphor and provides a number of useful extensions that facilitate the finding and adaptation of a complete and efficient course of action.

\section{A. The $<I-N-C-A>$ Ontology}

In $<\mathrm{I}-\mathrm{N}-\mathrm{C}-\mathrm{A}>$, both processes and process products are abstractly considered to be made up of a set of Issues (I) which are associated with the processes or process products to represent potential requirements, questions raised as a result of analysis or critiquing [1], etc. They also contain Nodes (N) (activities in a process, or parts of a physical product) which may have parts called sub-nodes making up a hierarchical description of the process or product. The nodes are related by a set of detailed Constraints (C) of various kinds. Finally there can be Annotations (A) related to the processes or products, which provide rationale, information and other useful descriptions. For a more detailed description of these four components see [14].

$<\mathrm{I}-\mathrm{N}-\mathrm{C}-\mathrm{A}>$ models, which are generic descriptions of synthesis tasks, are intended to support a number of different uses: 
- for automatic and mixed-initiative generation and manipulation of plans and other synthesized artifacts and to act as an ontology to underpin such use;

- as a common basis for human and system communication about plans and other synthesized artifacts;

- as a target for principled and reliable acquisition of knowledge about synthesized artifacts such as plans, process models and process product information;

- to support formal reasoning about plans and other synthesized artifacts.

These cover both formal and practical requirements and encompass the requirements for use by both human and computer-based planning and design systems.

\section{B. I-X Process Panels}

I-X Process Panels constitute the primary user interface to an I-X application. A panel more or less directly reflects the $<\mathrm{I}-\mathrm{N}-\mathrm{C}-\mathrm{A}>$ ontology underlying the whole I-X system.

When used to describe processes, nodes are the activities that need to be performed in a course of action, thus functioning as the items in an intelligent to-do list. The other elements contain issues as questions remaining for a given course of action, information about the constraints involved and the current state of the world, and annotations or notes such as reports or the rationale behind items in the plan. The user interface to the I-X system, the I-X Process Panel, shows four main parts that reflect the four components of the $<\mathrm{I}-\mathrm{N}-\mathrm{C}-\mathrm{A}>$ ontology. They are labeled "Issues", "Activities", "State", and "Annotations", as shown in figure 1.

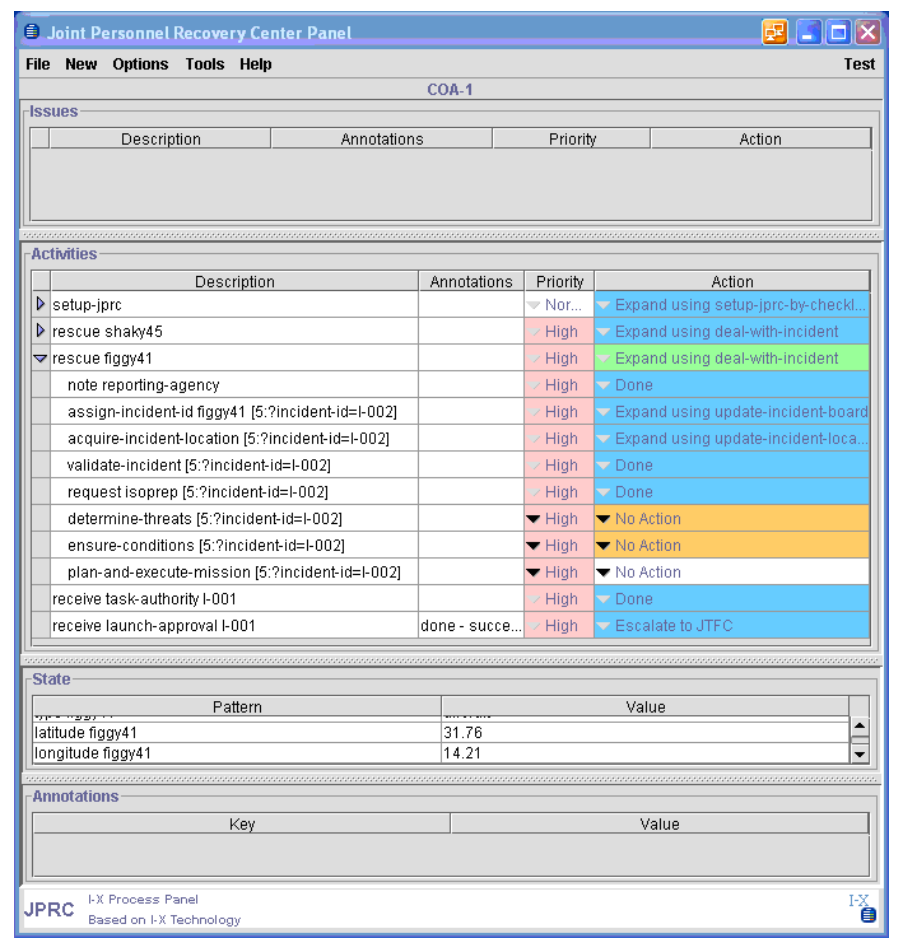

Figure 1: An I-X Process Panel.

\section{The Co-OPR PRojeCt AND APPLICATION}

The aim of the Co-OPR application was to support trainers and trainees in an emulated half day round of a CPX for a fictitious operation called Operation Able Sword. Such exercises were observed by the project team and researchers in October 2005, and materials were provided to enable research and experimentation.

\section{A. Command Post Exercises}

Command Post Exercises are performed at the PRETC as part of the PR course. The course consists of classroom teaching sessions and the CPX in which students are divided into groups, playing the roles of rescue centers that have to respond to some incidents that are emulated by the trainers.

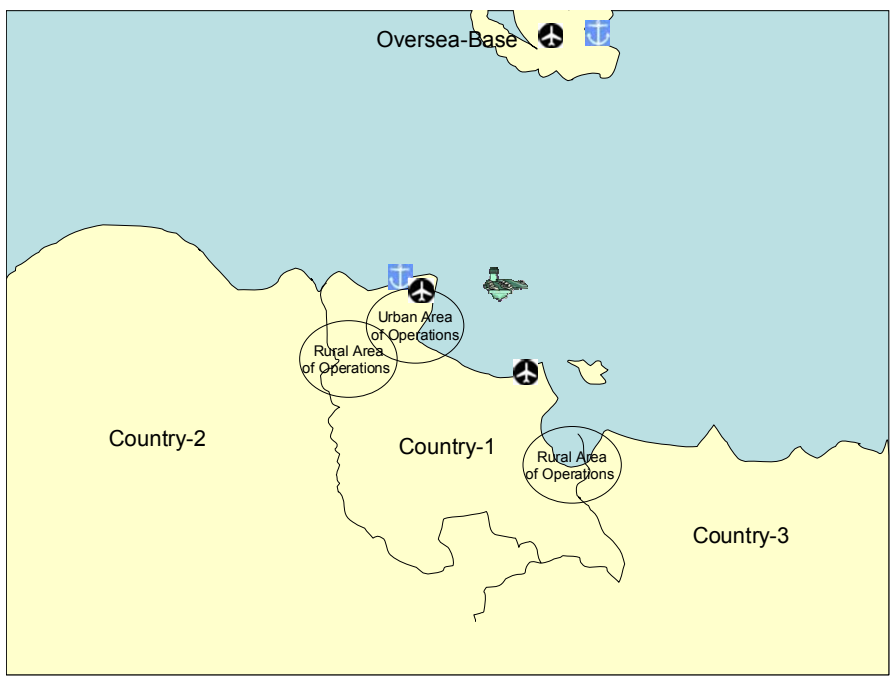

Figure 2: Generic Scenario Map

The context for the incidents and rescue missions that need to be launched is Operation Able Sword which nominally takes place in Tunisia on some given dates in June/July 2005. The topology corresponds to the generic map shown in figure 2 . In the figure, Country- 1 represents the country that is being assisted (Tunisia) and that is in conflict with its immediate neighbors. A shared coastline makes the involvement of the Navy possible. Country- 1 also has rural as well as urban areas that make for an interesting variety of potential incidents. Finally, a neutral country provides some oversea base that may play a role.

For a CPX, the students are divided into four groups and placed in different rooms where they act out the activities performed by the different Rescue Component Centers (RCC). In the CPX the Joint Personnel Recovery Center (JPRC) is colocated with the Air Force RCC. All other agents are roleplayed by the trainers at the PRETC. An overview of the organizational relationships between the different agents is given in figure 3. The first task for the students always consists of setting up the RCCs. Once this is completed the trainers call in incident reports to the different RCCs that have to be collected, analyzed and acted upon, usually by launching an appropriate rescue mission. 


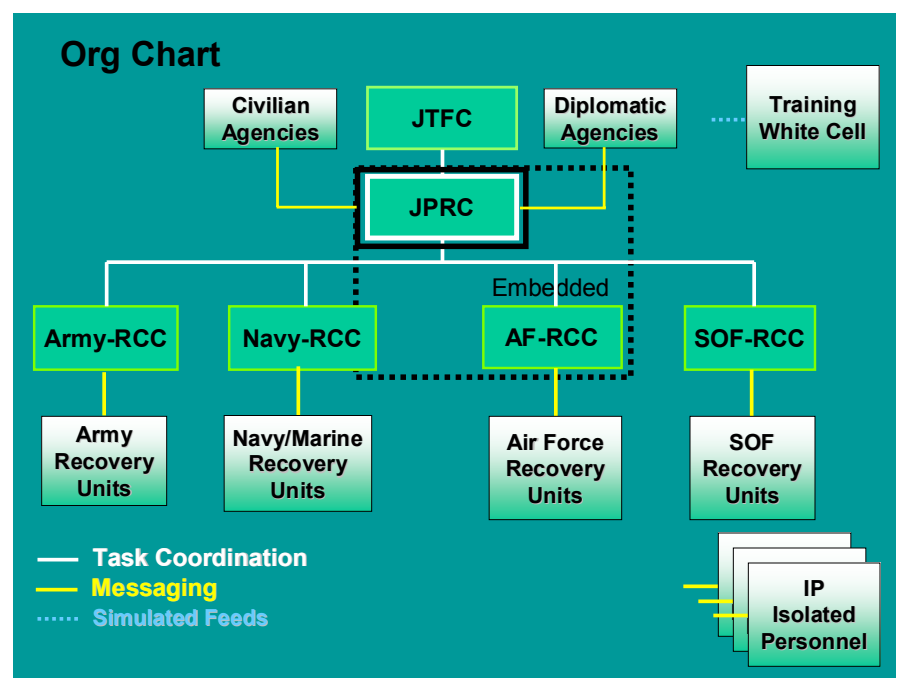

Figure 3: Organization of Agent in the Scenario

\section{B. I-X for the Co-OPR Application: Requirements}

In observing the Command Post Exercises at the PRETC, we have identified a number of ways in which I-X technology and the user interfaces or tools we can provide may be able to support those involved in search and rescue. I-X uses in a JPRC/RCC could include:

- Communications

- Simple Chat

- Structured chat

- Information sharing

- Task Support

○ Checklists

- To do list

○ Progress reporting

- Plan option aids

- Whiteboards
$\circ$ Incident
○ Weather/Codes/Info
- Assets

- Mapboards
- Terrain and GIS features
- Routes, ROZs, etc.
- Town and road plans
- Sketch maps

- Web Resources

○ Fact Book

- Phone List

o Codes

- Mission Folders

○ Attachments

Many of these features are already supported in the I-X framework generically. However, the JPRC and RCCs make heavy use of wall mounted whiteboards, maps, overlays on maps, and pin board material such as codes, phone lists, etc. We have implemented whiteboard and map orientated "viewers" that can all simultaneously share the same state in a single panel for display and sharing. We are now exploring ways in which the state underlying specific views can easily be shared with other users and I-X panels, and ways in which variances between the incoming and current believed state on any panel can be highlighted, such that the changes can initiate issues, activities, constraints or notes that need to be incorporated into the local plan.

We have also created a "white cell" support panel to assist the trainers in a CPX. This will allow:

- Driving a simulation of the world in which the training takes place, including starting and stopping moving assets such as fuel tankers, trucks, planes and ships.

- Setting the world clock as seen by all other I-X panels and users to a simulated time.

- Allowing master scenario event lists (MSELs) to be input and assist in driving the simulation

- Assisting in logging, noting training issues for report back, etc.

All these features are now part of the I-X framework and can be included in any I-X application. The first application to use them is the Co-OPR application described next.

\section{The Co-OPR Application}

The first step in developing an I-X application consists of deciding which agents to support. For the Co-OPR application it was clear that the most important agent is the JPRC which coordinates the efforts of the different RCCs. Two roles in the JPRC of particular importance are that of the director, who has to manage the centre and make sure everything that needs to be done gets done, and the controller who manages the recovery assets and has to come up with plans for individual recovery missions. Two I-X Process Panels were used to support these two roles. Only the second of these, the one for the controller, had the I-X option management facility enabled which can be used to explore possible courses of action and compare different recovery plans (see figure 4). Other RCCs were supported by a single panel only.

Another agent that plays an important role in the training scenario is the "white cell" that drives the scenarios and simulates the events that lead to the incidents the JPRC has to deal with. An I-X Process Panel was used to support this role by allowing for an additional communication channel with the other agents supported by panels. Finally, some other agents that play only minor roles in the different scenarios were included, e.g. the Joint Task Force Commander (JTFC) that has to give authorization for certain missions. The organization of all the agents in the application is as shown in figure 3.

To implement the task support it was necessary to model a set of standard operating procedures that could be used as refinements in the I-X Process Panel as described above. The refinements used were derived from two sources. Firstly, the U.S. manual for PR [4] was used as a base for knowledge engineering. Secondly, the checklists used by the PRETC during a CPX were imported into I X using a model import facility and manually updated in the I X Domain Editor. 


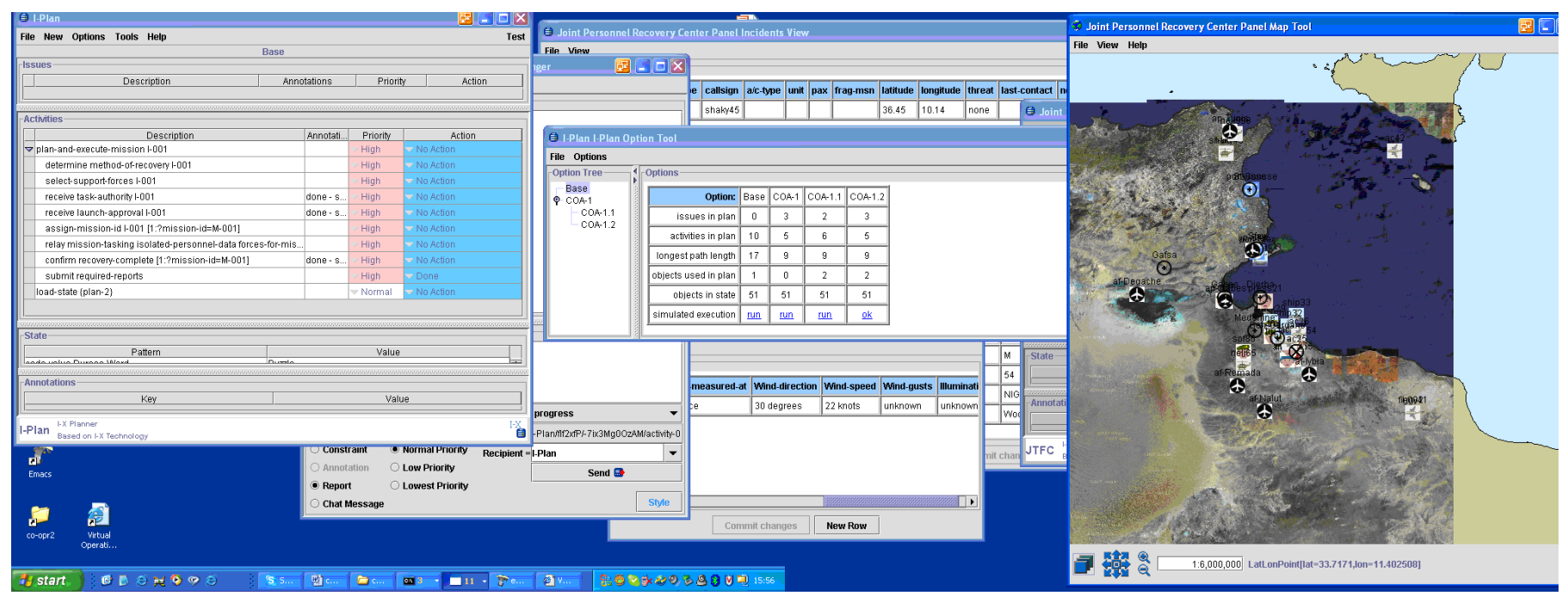

Figure 4: I-Plan Panel with plan completed; Option Tool with tree structure and comparison matrix; Map Tool showing locations

\section{EXPERIMENTS AND EVALUATION}

The experimentation was designed to demonstrate and stress the value of $\mathrm{I}-\mathrm{X}$ technology components in response to various individual events in sample incidents and missions provided by the PRETC. Following a number of progressively more realistic trials held in AIAI's experimental Emergency Response Coordination Center, two Co-OPR evaluation experiments were conducted in May and October 2006.

The experiments covered setting up a JPRC which is colocated with an Air Force RCC. Next, incidents of various kinds are dealt with, and a final operation is to prepare a shift change briefing. The aim of the experiment was to allow for an evaluation of the I-X technology as a support tool for both trainers and trainees. At this stage the evaluation was performed by Dr. Hansberger who was remotely observing the experiments from USJFCOM/J9. It is hoped that an evaluation with real users can take place later in the project.

The initial evaluation focused on the cognitive tasks that the JPRC director and JPRC controller performed when working in tandem to respond to the incidents that came into the JPRC as an emergency response coordination centre. This evaluation was necessarily limited in that, without a corresponding analysis of the performance with and use of the current in-situ systems and (manual) processes, a comparative assessment of the influence and worth of the I-X system as a whole is not possible. However, an analysis of the results throws up some interesting insights.

\section{A. Evaluation Methodology}

The evaluation methodology was straightforward. The director and the controller roles were played by two members of the I-X development team. In addition to being familiar with the use of I-X systems and with its deployment for this particular domain, these two have gained a basic competence in the objectives, approaches and working practices of the JPRC through observation and completion of basic training courses. An independent observer, a non-participant in the exercise (and also a member of the I-X team), was to observe their behaviour (aided and augmented by self-reporting by the subjects), determine the nature of the task that was currently being performed and the time at which the task began and ended, plus any additional comments or observations. In addition, the exercise was being video-taped, which would allow a retrospective analysis, perhaps with the assistance of the 'director' and 'controller', of any points during the exercise where the precise nature of the immediate task in hand was not clear. Importantly, the experiment was also observed by a member of the sponsoring organization familiar with PR and with systems evaluation. This was done remotely using Internet collaboration and desktop sharing tools including video teleconferencing.

Once this was done, in an attempt to generalize the various tasks that had been performed where appropriate each task was classified into one of several course-grained 'cognitive categories', namely:

information-gathering: these tasks involved searching for information that was required before the overall activity of the JPRC could be moved forward. In certain cases, this may involve looking up information in on-line databases, or paper-based manuals, or it may involve, say, (simulated) phonecalls to appropriate colleagues.

sense-making: these tasks involved an analysis and interpretation of information with the aim of understanding the problem, enumerating the different options that were available, listing the pros and cons of possible courses of action, and so on.

decision-making: these tasks involved the subject making a clear choice from among competing possible activities that would serve to achieve the objectives of the JPRC by effecting activity in other agents and then enacting this activity. So, for example, deciding to send a rescue helicopter to a particular destination and issuing the appropriate orders would be an 
example of a decision point, whereas deciding to look at a map would not, since it has no affect on other agents (and, instead, would probably be an instance of information-gathering).

housekeeping: these tasks involved the initial set-up of the JPRC environment, documentation of decisions, logging of calls, etc.

The first three of these categories (the housekeeping category being an artifact arising from the need to manage the JPRC and the 'paperwork' it generates) emerge from consideration of several different 'best practice' approaches to command and control and decision-making in general. For instance, Boyd's well-known OODA loop [8]-Observe, Orient, Decide, Act-can be seen to correspond with these three tasks, observe, orient, and decide. Observation is essentially synonymous in this context with informationgathering and orient is synonymous with sense-making. Since most of the decisions taken by the JPRC staff are done by issuing commands to others (i.e., in I-X terms, sending an activity to another agent) and this is done on the click of a mouse button, we do not attempt to differentiate the decide and act activities for our analysis. We instead conflate these two OODA tasks into the single decision-making category. Similarly, Wohl's SHORe (Stimulus, Hypothesis, Option, Response) framework [16] can be seen as analogous to our categories, with stimulus (Wohl's shorthand term for the information correlation and fusion phase) corresponding to information-gathering, hypothesis (Wohl's situation analysis phase) corresponding to sense-making, and the option and response phases being conflated into the single decisionmaking task (and for the same reason outlined above).

\begin{tabular}{|c|c|c|c|}
\hline Phase & OODA & SHORe & $\begin{array}{c}\text { "JPRC Experiment } \\
\text { C" Analysis }\end{array}$ \\
\hline 1 & observe & stimulus & $\begin{array}{c}\text { information- } \\
\text { gathering }\end{array}$ \\
\hline 2 & orient & hypothesis & sense-making \\
\hline 3 & decide & option & \multirow{2}{*}{ decision-making } \\
\hline 4 & act & response & \\
\hline
\end{tabular}

Table 1. Comparison of different Command-and-Control frameworks as they apply in this context; only part of the act (OODA) and response (SHORe) activities occurs within the context of the JPRC.

The correspondence between these different models is summarized in Table 1. The fundamental concept underlying all of these models is that a methodical approach to each cycle of the command and control 'loop', based on assembling information, interpreting that information, appraising possible courses of action and making and enacting decisions should lead to clear, consistent, and - ultimately - correct behaviour in situations where the pressure is great and time is short. Our empirical hypothesis here is that the use of the I-X system can encourage its users to adopt such a methodical approach to their task.

\section{B. Evaluation Results}

A fragment of the task analysis performed on the activities observed during Experiment $\mathrm{C}$ can be seen in figure 5 .

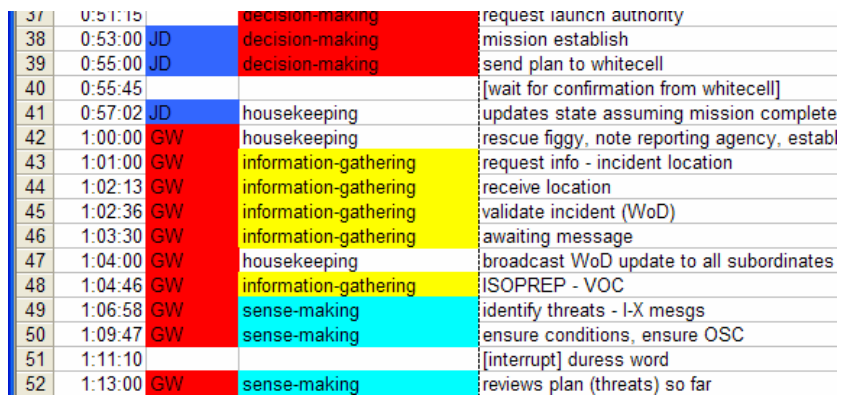

Figure 5: Fragment of Co-OPR task analysis.

Notwithstanding the provisos noted above about the inability at the time of writing to perform a full comparative evaluation, the analysis is encouraging for the use of the I-X in this task. In general, the use of SOPs encouraged a methodical approach to the overall JPRC activity: instances of information-gathering where followed by instances of sensemaking which led to decision-making episodes, with no instances of, for instance, a decision-making activity being interrupted or abandoned due to the lack of a crucial piece of information. In addition, at several times during the exercise, important messages arrived which interrupted the current activity and diverted the cognitive attention of the director or controller. Such interruptions can serve to disrupt the flow of the Center, but in the majority of cases, the framework provided by the SOPs allowed a quick resumption of activity once the message had been dealt with.

In addition, the analysis highlighted some areas where further support might prove helpful. In addition to dealing with interruptions, the arrival of new information that demands that the decisions made earlier in the process need to be re-appraised (and, in one case during the experiment, wholly abandoned, with rescue resources 'recalled') is currently not difficult to handle using within the SOP framework (and would seem to require something akin to 'exception-handling' procedures). Successfully dealing with such situations seems to rely heavily on the experience and initiative of the human in question. This would seem to be a general problem with any SOP-based system rather than with I-X per se, but technology that can offer more support would obviously be of great benefit.

Consideration of the time devoted during the experiment to each of the task categories is also interesting. While roughly the same amount of time was spent in information-gathering, sense-making and decision-making during the exercise, a surprisingly large amount of time was spent housekeeping twice as long, in fact, as the time spent for any of the other categories. This is due, in part, to the time required to initialize the JPRC and check that its procedures and communications are in place, and then later to produce a report summarizing the session activities for the next duty officer. Providing automated assistance for these tasks may reduce the workload 
of the humans involved while also ensuring a more rapid and efficient establishment of the Center and hand-over of duty.

Aside from an analysis of the cognitive tasks performed by the system users, the experimentation also highlighted a number of open issues with the current prototype. Firstly, support for the white cell was rather limited at this stage. Only the structured messaging feature was a real advantage provided by I-X. However, the way the scenario was driven was adapted to this way of delegating tasks, which does not correspond well to the way the real CPX works. This in effect removes a large part of the sense-making task from the problem and shifts the focus onto the planning activities, an area in which I-X is strong. Secondly, the two panels used by the director and the controller are equipped with independent $<\mathrm{I}-\mathrm{N}-\mathrm{C}-\mathrm{A}>$ models which may lead to inconsistent world state representations within the JPRC. While this did not occur during the experiment, it is a potential problem that was noted. Finally, some issues with the user interface need to be addressed for future versions, e.g. the lack of a mechanism to draw the user's attention immediately to new, incoming activities.

\section{CONCLUSIONS}

The aim of the Co-OPR experimentation was to emulate a half-day round of a CPX usually held at the U.S. Personnel Recovery Education and Training Center. Materials were provided by DARPA and USJFCOM for a search and rescue element of a military mission. The experiments were designed to demonstrate and stress the I-X technology components in response to various individual events in sample training missions and events provided by experience trainers in the US Joint Personnel Recovery Agency.

Initial evaluation indicates that $\mathrm{I}-\mathrm{X}$ can indeed be used to build applications that support task-centric activities in this domain, and that two main features supported by I-X, namely intelligence through integrated standard operation procedures, and coordination support through linked process panels, are useful in supporting the overall activity of a Coalition or Joint Personnel Recovery Center. More specifically, an analysis of the experiment shows that the hierarchical structure of the tasks in the to-do list helps users to focus their efforts and avoid distractions, and if interrupted, it helps them to quickly continue with important decision making without having to repeat information-gathering or sense-making activities that have already been completed. It can also help in handover between personnel when staffs change as it presents a clear status picture of tasks and actions that have been accomplished in the past, on-going current activities, and future tasks needing completion.

\section{ACKNOWLEDGMENT}

The I-X project is sponsored by the Defense Advanced Research Projects Agency (DARPA) under agreement number F30602-03-2-0014. The University of Edinburgh, the U.S. government and research sponsors are authorized to reproduce and distribute reprints and on-line copies for their purposes notwithstanding any copyright annotation hereon. The views and conclusions contained herein are those of the authors and should not be interpreted as necessarily representing the official policies or endorsements, either expressed or implied, of other parties.

\section{REFERENCES}

[1] Buckingham Shum, S., Selvin, A., Sierhuis, M., Conklin, J., Haley, C. and Nuseibeh, B. (2006). Hypermedia Support for Argumentation-Based Rationale: 15 Years on from gIBIS and QOC. In: Rationale Management in Software Engineering (Eds.) A.H. Dutoit, R. McCall, I. Mistrik, and B. Paech. Springer-Verlag: Berlin

[2] Conklin, J. (2003) Dialog Mapping: Reflections on an Industrial Strength Case Study. In: P.A. Kirschner, et al. (eds.) Visualizing Argumentation: Software Tools for Collaborative and Educational Sense-Making. Springer-Verlag: London, pp. 117-136.

[3] Horling, B., Lesser, V., Vincent, R. and Wagner, T. (2006) The Soft Real-Time Agent Control Architecture. Autonomous Agents and MultiAgent Systems, Volume 12, Number 1, Springer Science+Business Media, pp. 35-92.

[4] Joint Publication 3-50 (2005) Joint Doctrine for Personnel Recovery, 2nd Draft, March 2005.

[5] Kreifelts, Th., Hinrichs, E., and Woetzel, G. (1993) Sharing To-Do Lists with a Distributed Task Manager. In: de Michelis, G. and Simone, C. (eds.) Proceedings of the 3rd European Conference on Computer Supported Cooperative Work, pp 31-46, Milano, 13-17 September 1993, Kluwer, Dordrecht.

[6] MacLean, A., Young, R., Bellotti, V. and Moran, T. (1991) Design space analysis: Bridging from theory to practice via design rationale. In Proceedings of Esprit '91, Brussels, November 1991, pp 720-730.

[7] Nau, D., Cao, Y., Lotem, A., Muñoz-Avila, H. (1999) SHOP: A Simple Hierarchical Ordered Planner. Proc IJCAI 1999, pp. 968-973.

[8] Osinga, F. (2006) Science Strategy and War, The Strategic Theory of John Boyd, Abingdon, UK: Routledge, ISBN 0-415-37103-1.

[9] Polyak, S. and Tate, A. (1998) Rationale in Planning: Causality, Dependencies and Decisions. Knowledge Engineering Review, Vol.13(3), pp 247-262.

[10] Selvin, A.M. (1999) Supporting Collaborative Analysis and Design with Hypertext Functionality, Journal of Digital Information, Vol. 1 Issue 4.

[11] Selvin, A.M., Buckingham Shum, S.J., Sierhuis, M., Conklin, J., Zimmermann, B., Palus, C., Drath, W., Horth, D., Domingue, J., Motta, E. and Li, G. (2001) Compendium: Making Meetings into Knowledge Events. Knowledge Technologies 2001, Austin TX, USA, pp 4-7.

[12] Tate, A. (1995) Integrating Constraint Management into an AI Planner. Journal of Artificial Intelligence in Engineering, Vol. 9, pp 221-228.

[13] Tate, A., Dalton, J. and Stader, J. (2002) I-P2- Intelligent Process Panels to Support Coalition Operations. In Proceedings of the Second International Conference on Knowledge Systems for Coalition Operations (KSCO-2002). Toulouse, France, April 2002.

[14] Tate, A. (2003) <I-N-C-A>: An Ontology for Mixed-initiative Synthesis Tasks. Proceedings of the Workshop on Mixed-Initiative Intelligent Systems (MIIS) at the International Joint Conference on Artificial Intelligence (IJCAI-03), Acapulco, Mexico, August 2003, pp 125-130.

[15] Wickler, G., Tate, A. and Potter, S. (2006) Using the <I-N-C-A> Constraint Model as a Shared Representation of Intentions for Emergency Response, Proc. 1st Int. Workshop on Agent Technology for Disaster Management (ATDM), at the 5th Int. Joint Conference on Autonomous Agents and Multiagent Systems (AAMAS 2006), Hakodate, Japan, May 8-12, 2006.

[16] Wohl, J.G. (1981) Force Management Decision Requirements for Air Force Tactical Command and Control. IEEE Transactions on Systems, Man, and Cybernetics, 1981, 11. 